\title{
MicroRNA-124-3p directly targets PDCD6 to inhibit metastasis in breast cancer
}

\author{
LING ZHANG ${ }^{1,2}$, XIANGMING CHEN ${ }^{2}$, BAOLI LIU ${ }^{3}$ and JUNQING HAN ${ }^{1}$ \\ ${ }^{1}$ Department of Tumor Research and Therapy Center, Shandong Provincial Hospital Affiliated to \\ Shandong University, Jinan, Shandong 250000; Departments of ${ }^{2}$ Clinical Oncology and \\ ${ }^{3}$ Medical Insurance, Taian City Central Hospital, Taian, Shandong 271000, P.R. China
}

Received March 26, 2016; Accepted August 23, 2017

DOI: $10.3892 / \mathrm{ol} .2017 .7358$

\begin{abstract}
Breast cancer (BC) is the leading cause of cancer-associated mortality among women worldwide, with a poor 5-year survival rate, particularly among patients with metastatic BC. Previous studies have indicated that the dysregulation of microRNAs (miRNAs/miRs) is associated with carcinogenesis and metastasis. Thus, investigating the underlying molecular mechanisms by which miRNAs mediate their effects may aid in the improvement of $\mathrm{BC}$ treatment. In the present study, reverse transcription-quantitative polymerase chain reaction analyses were performed to investigate miR-124-3p expression in BC tissues. The expression of miR-124-3p was significantly decreased in primary BC tissues compared with that in adjacent non-tumor tissues. Downregulated miR-124-3p was correlated with lymph node metastasis and a low overall survival time. Wound-healing and Transwell assays revealed that MDA-MB-231 and MCF-7 cell motility was inhibited by miR-124-3p, but was promoted by a miR-124-3p inhibitor. Overexpression of miR-124-3p increased levels of E-cadherin, and decreased levels of $\mathrm{N}$-cadherin and Vimentin, indicating that miR-124-3p inhibits the epithelial-mesenchymal transition. In addition, a bioinformatics analysis and subsequent in vitro experiments identified programmed cell death protein 6 (PDCD6) as a direct target of miR-124-3p. Restoration of PDCD6 expression impaired the metastasis inhibitor role of miR-124-3p by promoting cell invasion. Furthermore, the expression of miR-124-3p was inversely associated with PDCD6 mRNA levels in clinical breast tumors. Taken together, these data suggest that miR-124-3p inhibits tumor metastasis by inhibiting PDCD6 expression, and that the miR-124-3p/PDCD6 signaling axis
\end{abstract}

Correspondence to: Professor Junqing Han, Department of Tumor Research and Therapy Center, Shandong Provincial Hospital Affiliated to Shandong University, 324 Jingwu Road, Jinan, Shandong 250000, P.R. China

E-mail: hanjunqing1960@163.com

Key words: microRNA-124-3p, metastasis, PDCD6, epithelialmesenchymal transition, breast cancer may be a potential target for novel treatments in patients with advanced BC.

\section{Introduction}

Breast cancer (BC) is the most common malignancy and the first leading cause of tumor-associated mortality in females worldwide (1), and the frequency of $\mathrm{BC}$ is increasing in developed and developing countries (2). Tumor metastasis is the primary reason for the poor prognosis or high mortality of $\mathrm{BC}$ (3), therefore the identification of biomarkers for tumor metastasis may aid in developing novel treatments for, and in the diagnosis of, $\mathrm{BC}$.

MicroRNAs (miRNAs/miRs) are a class of small, non-coding, single-stranded RNAs that regulate multiple target mRNAs, generally through perfect or imperfect binding to the 3'-untranslated region (UTR) of the mRNA. Previous studies have indicated that miRNAs serve critical roles in various tumor-associated biological processes, including proliferation, metastasis, apoptosis and differentiation (4-6). Dysregulation of miRNAs leads to a tumor suppressive or oncogenic effect depending on the target mRNAs, and ultimately contributes to the initiation and progression of human malignancies (7-9). Therefore, miRNAs may have potential as diagnostic or prognostic biomarkers for cancer.

In the present study, miR-124-3p was investigated as a metastasis inhibitor and its expression in $\mathrm{BC}$ tissues was determined. PDCD6 was also investigated as target of miR-124-3p. This data expands our knowledge of the role of miR-124-3p in $\mathrm{BC}$ development and considers the miR-124-3p/PDCD6 signaling axis as an important underlying molecular mechanism and effective therapeutic strategy for advanced BC.

\section{Materials and methods}

Tissue specimens and cell lines. Primary $\mathrm{BC}$ tissues and corresponding non-tumor tissues were obtained during surgery from 40 female patients (mean age, 54.52 years; range, 42-68 years) who were diagnosed and treated at Shandong Provincial Hospital Affiliated to Shandong University (Jinan, China) between February 2010 and August 2011. Tumor stage was recorded according to the classification criteria of the Union for International Cancer Control (10). All tumors and 
normal tissues were confirmed by two independent pathologists. Matched non-cancerous tissues from the macroscopic tumor margin were obtained and used as normal controls. The clinicopathological characteristics were obtained from medical records. The present study was approved by the Ethics Committee of Shandong Provincial Hospital and written informed consent was obtained from patients prior to surgery.

Human BC MCF-7 and MDA-MB-231 cells were purchased from the American Type Culture Collection (Manassas, VA, USA), and cultured at $37^{\circ} \mathrm{C}$ with $5 \% \mathrm{CO}_{2}$ in a humidified incubator. The MCF-7 cells were cultured in RPMI-1640 medium, and the MDA-MB-231 cells were cultured in Dulbecco's modified Eagle's medium (both Gibco; Thermo Fisher Scientific, Inc., Waltham, MA, USA) supplemented with $10 \%$ fetal bovine serum (FBS; Gibco; Thermo Fisher Scientific, Inc.).

Reverse transcription-quantitative polymerase chain reaction $(R T-q P C R)$ analysis of miRNA and $m R N A$ levels. RT-qPCR assays were performed to determine the expression level of miR-124-3p and PDCD6 mRNA in cells or tissues. Total RNA was extracted using TRIzol reagent (Invitrogen; Thermo Fisher Scientific, Inc.), according to the manufacturer's protocol, and the RNA concentration was measured using a NanoDrop ${ }^{\text {TM }} 2000$ spectrophotometer (Thermo Fisher Scientific, Inc., Wilmington, DE, USA). For miRNA detection, total RNA was used to generate complementary (c)DNA using the PrimeScript RT reagent kit (Clontech Laboratories, Inc., Mountainview, CA, USA), and the SYBR ${ }^{\circledR}$ Premix ExTaq (Clontech Laboratories Inc.) was used to perform RT-qPCR. For mRNA detection, $500 \mathrm{ng}$ total RNA was subjected to first-strand cDNA synthesis using M-MLV Reverse Transcriptase (Promega Corporation), and RT-qPCR assay was performed using the SYBR-Green PCR master mix (Roche Diagnostics, Basel, Switzerland) on the Fast Real-Time ABI 7500 PCR System (Applied Biosystems; Thermo Fisher Scientific, Inc.). The following PCR conditions were as follows: $95^{\circ} \mathrm{C}$ for $30 \mathrm{sec}$, followed by 42 cycles at $95^{\circ} \mathrm{C}$ for $30 \mathrm{sec}, 60^{\circ} \mathrm{C}$ for $30 \mathrm{sec}$ and $72^{\circ} \mathrm{C}$ for $30 \mathrm{sec}$. The sequences of the primers were as follows: PDCD6 (sense), 5'-CGGCAA GTTGTCGGAGACG-3'; PDCD6 (antisense), 5'-CCTGGA GGTTGGGATGCTCT-'; $\beta$-actin (sense), 5'-AGGGAAATC GTGCGTGAC-3'; $\beta$-actin (antisense), 5'-CGCTCATTGCCG ATAGTG-3'; miR-124-3p (sense), 5'-TAAGGCACGCGGTGA ATGCC-3'; miR-124-3p (antisense), 5'-GATTGAATCGAG CACCAGTTAC-3'; U6 (sense), 5'-CGCTTCGGCAGCACA TATACTA-3'; U6 (antisense) 5'-GATTGAATCGAGCAC CAGTTAC-3'. The relative expression of miR-124-3p and PDCD6 mRNA was normalized to U6 spliceosomal RNA and $\beta$-actin, respectively, using the $2^{-\Delta \Delta \mathrm{Cq}}$ method (11). RT-PCR was performed in triplicate.

Transfection and plasmid construction. miR-124-3p mimics (5'-UAAGGCACGCGGUGAAUGCC-3'), inhibitor (5'-UAA GGCACGCGGUGAAUGCC-3'), mimic negative control (con M; 5'-UUCUCCATCGUGCCUCUAT-3'), and inhibitor negative control (con I; 5'-CCGUACUUCGCUAGAUCA-3') were synthesized by Guangzhou RiboBio Co., Ltd. (Guangzhou, China) and transfected into cells using Lipofectamine ${ }^{\circledR} 2000$ (Invitrogen; Thermo Fisher Scientific, Inc.) according to the manufacturer's protocol. For the luciferase reporter assay, the
3'-UTR of PDCD6 containing the putative binding sites for miR-124-3p was amplified from genomic DNA by PCR and cloned into the pGL3-luciferase reporter plasmid (Promega Corporation, Madison, WI, USA). The Platinum ${ }^{\mathrm{TM}}$ Taq DNA Polymerase (Invitrogen; Thermo Fisher Scientific, Inc.) was used in PCR reaction and conditions were as followed: $95^{\circ} \mathrm{C}$ for $1 \mathrm{~min}$, followed by 45 cycles of $95^{\circ} \mathrm{C}$ for $30 \mathrm{sec}, 56^{\circ} \mathrm{C}$ for $20 \mathrm{sec}$ and $72^{\circ} \mathrm{C}$ for $20 \mathrm{sec}$. The primers used to amplify the 3'-UTR of PDCD6 were as follows: Forward, 5'-GCTACC TGACTAAGCAT-3' and reverse, 5'-CCTAGATCGGATAGC TAGC-3'. Mutations in the binding site of the PDCD6 3'-UTR were generated using the QuikChange ${ }^{\mathrm{TM}}$ Site-Directed Mutagenesis kit (Agilent Technologies, Inc., Santa Clara, CA, USA) according to the manufacturer's protocol. The sequence lacking the PDCD6 3'-UTR was introduced into cells to rescue PDCD6 expression using pcDNA3.1(+) (Invitrogen; Thermo Fisher Scientific, Inc.) plasmid. The primers used to amplify PDCD6 without the 3'-UTR were as follows: Forward, 5'-TGC GTAGCCTAGTACAG-3' and reverse, 5'-GTCGACTGACAG GCTA-3'.

Wound-healing and Transwell assays. To determine the effect of upregulated or downregulated miR-124-3p on cell migratory behavior, a wound-healing assay was performed and the closure of the wounds in each well was evaluated. The two cell lines were seeded onto $35-\mathrm{mm}$ dishes coated with fibronectin, and the cells were allowed to reach $100 \%$ confluency. An artificial wound was created on a confluent cell monolayer without FBS using a 200-ml pipette tip $24 \mathrm{~h}$ after transfection. The culture medium was refreshed with serum-free medium, and the cells were incubated for additional $24 \mathrm{~h}$ at $37^{\circ} \mathrm{C}$. Serial images were obtained at 0 and $24 \mathrm{~h}$ using a light microscope (Olympus, Tokyo, Japan).

For the invasion assay, $2 \times 10^{4}$ transfected cells were seeded into the upper chamber of the Matrigel ${ }^{\circledR}$ Transwell chamber (BD Biosciences, Franklin Lakes, NJ, USA). Medium containing $10 \%$ FBS in the lower chamber acted as the chemoattractant. Following incubation for a further $48 \mathrm{~h}$ at $37^{\circ} \mathrm{C}$, non-invading cells were removed from the upper surface with a cotton swab, and the invaded cells attached to the lower surface of the membrane were fixed with methanol, stained with $0.1 \%$ crystal violet and then counted using an Olympus light microscope (Olympus Corporation, Tokyo, Japan).

Western blotting analysis. Western blotting analysis was performed according to a standard protocol. Briefly, the cells were washed twice with ice cold PBS and lysed on ice in RIPA buffer (Beyotime Institute of Biotechnology, Haimen, China) and the protein concentration was determined using the Pierce BCA protein assay kit (Thermo Fisher Scientific, Inc.). Equal amounts of proteins $(20 \mu \mathrm{g})$ were separated on a 10\% SDS-PAGE gel and then transferred to polyvinylidene fluoride membranes (EMD Millipore, Billerica, MA, USA). The membranes were blocked with $5 \%$ skimmed milk and incubated at room temperature for $2 \mathrm{~h}$ with an anti-PDCD6 antibody (catalog no. ab109181; dilution, 1:2,000; Abcam, Cambridge, MA, USA), anti-E-cadherin antibody (catalog no. ab15148; dilution, 1:500; Abcam), anti-N-cadherin antibody (catalog no. ab15148; dilution, 1:500; Abcam), anti-Vimentin antibody (catalog no. ab137321; dilution, 1:1,000; Abcam) and $\beta$-actin (catalog no. A5316; dilution, 
Table I. Clinicopathological characteristics of patients with breast cancer and correlation with miR-124-3p expression.

\begin{tabular}{|c|c|c|c|}
\hline Clinicopathological characteristic & No. of patients $(n=40)$ & miR-124-3p level (mean \pm standard deviation) & P-value \\
\hline Age, years & & & $0.652^{\mathrm{a}}$ \\
\hline$<55$ & 23 & $0.0161 \pm 0.0074$ & \\
\hline$\geq 55$ & 17 & $0.0190 \pm 0.0103$ & \\
\hline Differentiation & & & $0.911^{\mathrm{b}}$ \\
\hline Well & 6 & $0.0124 \pm 0.0066$ & \\
\hline Moderate & 20 & $0.0179 \pm 0.0100$ & \\
\hline Poor & 14 & $0.0170 \pm 0.0081$ & \\
\hline Tumor stage & & & $0.192^{\mathrm{b}}$ \\
\hline $\mathrm{I}$ & 8 & $0.0189 \pm 0.0089$ & \\
\hline II & 8 & $0.0200 \pm 0.0053$ & \\
\hline III & 22 & $0.0163 \pm 0.0099$ & \\
\hline IV & 2 & $0.0118 \pm 0.0052$ & \\
\hline Lymph node & & & $0.006^{\mathrm{a}}$ \\
\hline$(-)$ & 17 & $0.0216 \pm 0.0098$ & \\
\hline$(+)$ & 23 & $0.0142 \pm 0.0064$ & \\
\hline Estrogen receptor status & & & $0.419^{\mathrm{a}}$ \\
\hline Negative & 26 & $0.0183 \pm 0.0093$ & \\
\hline Positive & 14 & $0.0155 \pm 0.0076$ & \\
\hline
\end{tabular}

${ }^{a}$ Mann-Whitney test; ${ }^{b}$ Kruskal-Wallis test. miR, miRNA.

1:5,000; Sigma-Aldrich; Merck KGaA, Darmstadt, Germany). Following washing with Tris-buffered saline with Tween-20 [10 mM Tris ( $\mathrm{pH} 8.0), 150 \mathrm{mM} \mathrm{NaCl}$ and $0.1 \%$ Tween-20], the membranes were then incubated with a secondary goat anti-rabbit horseradish peroxidase-conjugated antibody (catalog no. sc-2004; dilution, 1:5,000; Santa Cruz Biotechnology, Santa Cruz, CA, USA) for $1 \mathrm{~h}$ at $37^{\circ} \mathrm{C}$. The protein bands were detected using an enhanced ECL chemiluminescent Substrate kit (Pierce; Thermo Fisher Scientific, Inc.).

Dual luciferase reporter assay. Recombinant plasmids of pGL3-PDCD6-3'-UTR-wild-type (WT) or pGL3-PDCD6-3'-UTR-Mutation (Mut) were constructed as aforementioned. Cells $\left(1 \times 10^{5}\right.$ cells/well $)$ were seeded in a 24-well plate and co-transfected with $40 \mathrm{nM}$ of either miR-124-3p mimic or mimic negative control, $20 \mathrm{ng}$ of either pGL3-PDCD6-3'-UTR-WT or pGL3-PDCD6-3'-UTR-Mut, and $2 \mathrm{ng}$ of pRL-TK (Promega Corporation) using Lipofectamine 2000, according to the manufacturer's protocol. Following incubation for $48 \mathrm{~h}$, the cells were lysed and the luciferase reporter assay was performed using the Dual-Luciferase ${ }^{\circledR}$ Reporter assay system (Promega Corporation), according to the manufacturer's protocol.

Target prediction. To identify the potential targets of miR-124-3p, a bioinformatics analysis was performed using three publically available databases: TargetScan (http://www.targetscan.org/vert_71/), miRDB (http://www .mirdb.org/miRDB/) and microRNA (http://www.microrna .org/microrna/home.do). miRDB is an online database for miRNA target prediction and functional annotations.
Statistical analysis. All data were analyzed using SPSS software (version 13.0; SPSS, Inc., Chicago, IL, USA) or GraphPad Prism (version 5; GraphPad Software, Inc., La Jolla, CA, USA). The results obtained from in vitro assays are presented as the mean \pm standard error of the mean and the data were analyzed by Student's t-test. A paired samples t-test was performed to investigate the differences in miR-124-3p and PDCD6 mRNA expression between normal and cancerous tissues. A Mann-Whitney test was used to compare differences in miR-124-3p levels between two groups of patients with certain clinicopathological characteristics, while a Kruskal-Wallis test was performed to compare more than two groups, followed by Dunn's multiple comparison post-hoc test between groups. The correlation between miR-124-3p and PDCD6 was analyzed using a Spearman's rank correlation test. A Kaplan-Meier estimator analysis and a log-rank test were performed for the overall survival analysis. $\mathrm{P}<0.05$ was considered to indicate a statistically significant difference.

\section{Results}

miR-124-3p is downregulated in BC tissue compared with non-tumor tissue in patients with $B C$. To further study the association between miR-124 and BC pathogenesis, the expression of miR-124-3p in 40 patients with BC was detected using RT-qPCR. The expression of miR-124-3p was significantly decreased in $\mathrm{BC}$ tissues compared with the corresponding non-tumor tissues $(0.0174 \pm 0.0088$ vs. $0.0226 \pm 0.0139 ; \mathrm{P}=0.003$, paired t-test; Fig. 1A). In addition, the associations between the expression level of miR-124-3p and different clinicopathological characteristics of patients with BC were investigated. As illustrated in Table I, 
A

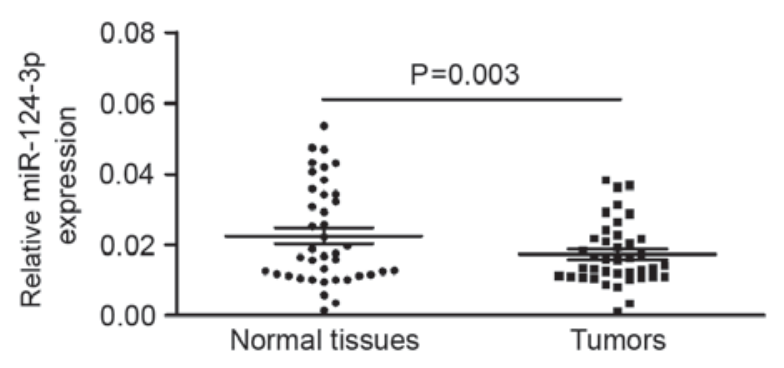

B

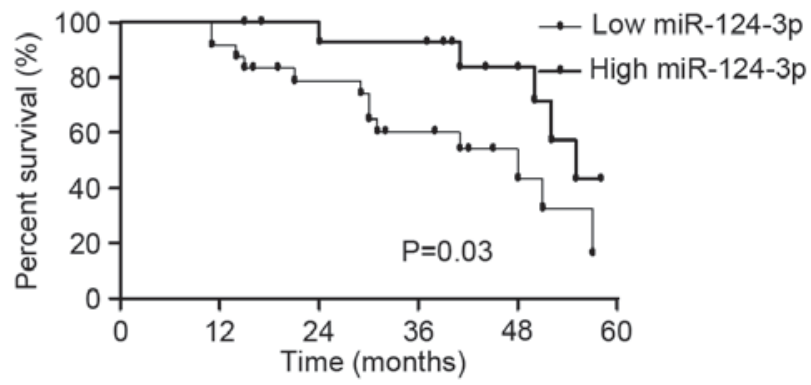

Figure 1. Expression of miR-124-3p is downregulated in primary tumors. (A) Expression of miR-124-3p quantified using reverse transcription-quantitative polymerase chain reaction in $\mathrm{BC}$ tissues and paired adjacent normal tissues from 40 patients. Data was normalized to U6 spliceosomal RNA expression. (B) Low expression of miR-124-3p predicts a poor prognosis for patients with BC. miR, miRNA; BC, breast cancer.

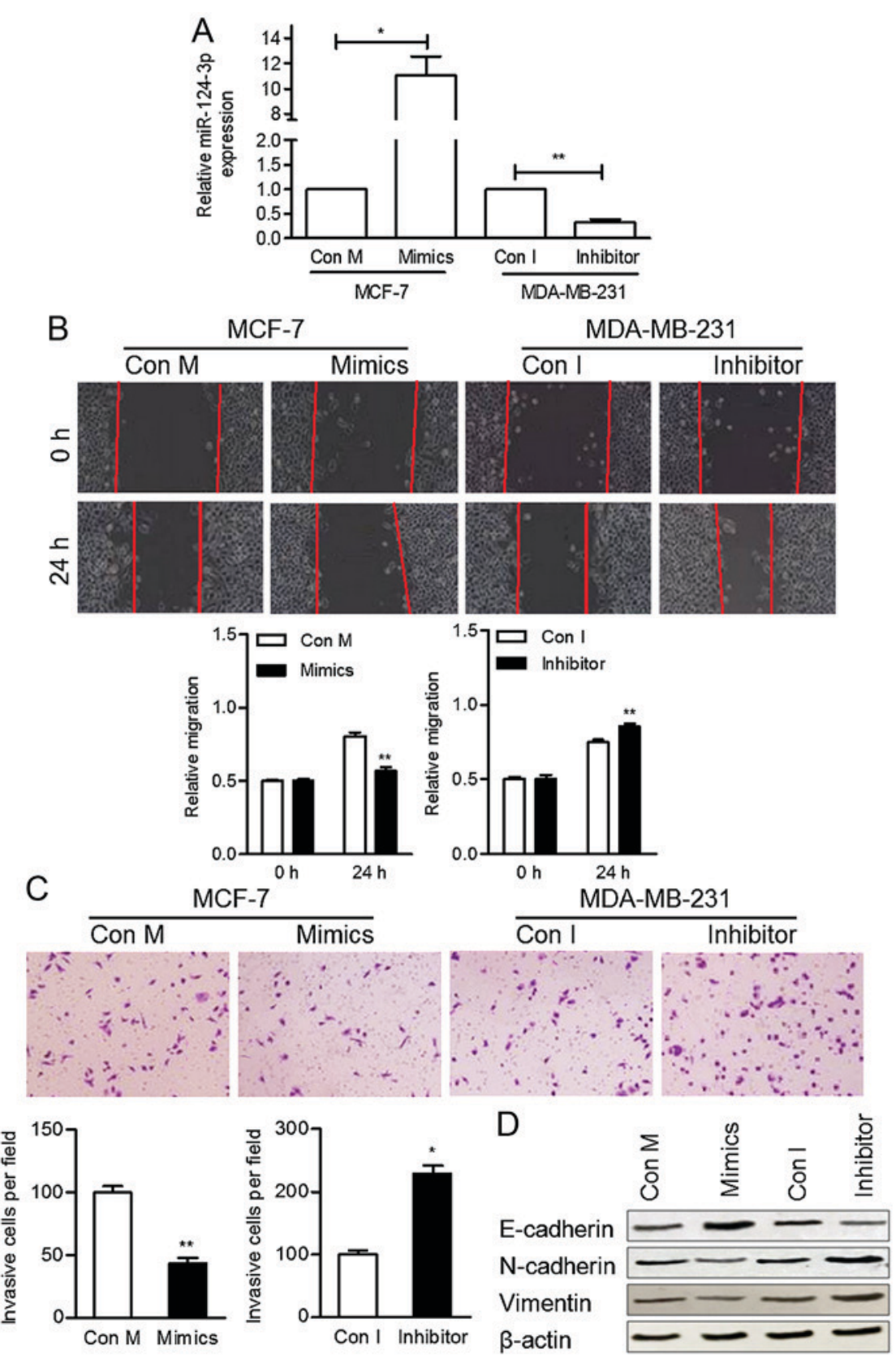

Figure 2. miR-124-3p inhibits BC cell migration and invasion. (A) Overexpression or downregulation of miR-124-3p was confirmed by reverse transcription-quantitative polymerase chain reaction. (B) Wound-healing and (C) Transwell assays demonstrated changes in cell migration and invasion, respectively; magnification, x200. (D) The protein levels of epithelial-mesenchymal transition-related markers were determined by western blotting analysis following transfection with miR-124-3p mimics or an inhibitor. " $\mathrm{P}<0.05$ and ${ }^{* *} \mathrm{P}<0.01$ vs. Con $\mathrm{M}$ and Con I. miR, miRNA; BC, breast cancer; Con M, mimics control; Con I, inhibitor control. 
A

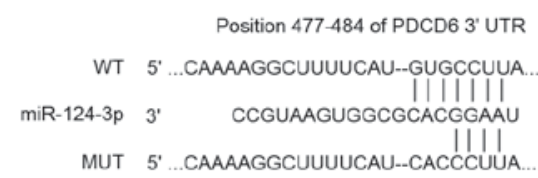

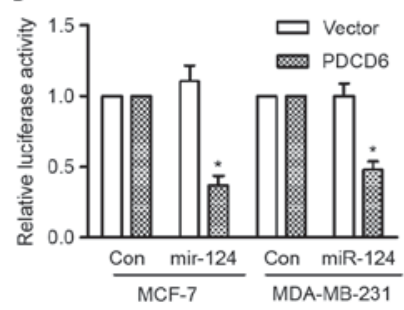

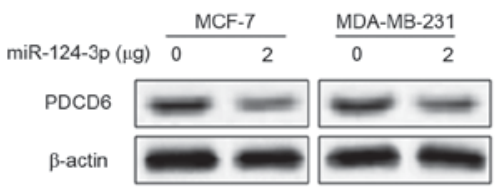

Figure 3. miR-124-3p targets PDCD6 3'-UTR mRNA. (A) The binding sites of PDCD6 mRNA 3'-UTR matching the seed sequence of miR-124-3p. (B) Dual-luciferase reporter assays in BC cells that were transiently transfected with the reporter plasmids with or without miR-124-3p mimics. (C) Western blotting analysis of PDCD6 protein levels in BC cells transfected with miR-124-3p $(2 \mu \mathrm{g})$ or a blank control $(0 \mu \mathrm{g})$. "P<0.05 vs. vector. miR, miRNA; PDCD6, programmed cell death protein 6; UTR, untranslated region; BC, breast cancer; WT, wild-type; MUT, mutant.
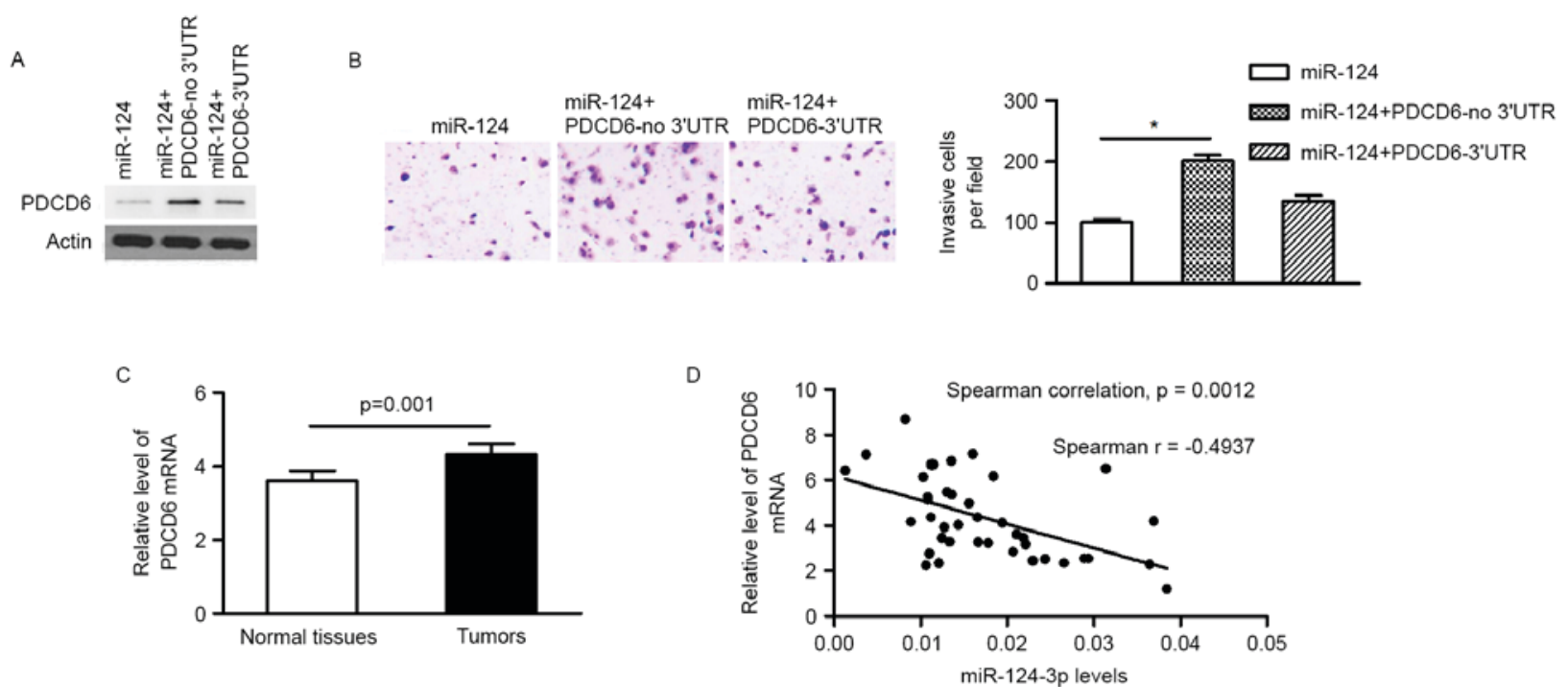

Figure 4. Overexpression of PDCD6 reverses the effect of miR-124-3p on MCF7 cells. (A) Reintroduction of PDCD6 expression by transfection of cells with a vectors containing full length PDCD6 or PDCD6 lacking a 3'-UTR. (B) Transwell invasion assays in MCF7 cells co-transfected and the vectors from (A). (C) The mRNA levels of PDCD6 were markedly increased in tumors compared with adjacent normal tissues. (D) miR-124-3p expression was negatively correlated with PDCD6 in tumor tissues from patients with BC. "P<0.05. PDCD6, programmed cell death protein 6; miR, miRNA; UTR, untranslated region; $\mathrm{BC}$, breast cancer.

the expression of miR-124-3p was markedly lower in patients with lymph node metastasis compared with that in patients without lymph node metastasis ( $\mathrm{P}=0.006$, Mann-Whitney test). When comparing other subgroups, including age $(<55$ and $\geq 55$ years old), differentiation (well/moderate/poor), tumor stage (I/II/III/IV), and estrogen receptor status (negative/positive), the differences in miR-124-3p levels were not statistically significant. The mean level of miR-124-3p expression in the BC tissues was used as a threshold to divide patients into two groups (low or high expression groups). Kaplan-Meier estimator analyses indicated that patients with BC with high miR-124-3p expression were likely to survive longer than those with low miR-124-3p expression (Fig. 1B).

miR-124-3p inhibits cell migration and invasion in vitro. As downregulated miR-124-3p expression was associated with lymph node metastasis, the effect of miR-124-3p on cell motility was investigated. miR-124-3p was overexpressed or inhibited by transfecting MCF-7 and MDA-MB-231 cells with miR-124-3p mimics and a miR-124-3p inhibitor, respectively. The expression of miR-124-3p in transfected cells was confirmed using RT-qPCR (Fig. 2A).
The migratory ability of these cells was subsequently determined using a wound-healing assay. MCF-7 cells treated with miR-124-3p mimics were significantly less migratory compared with the control cells at $24 \mathrm{~h}(\mathrm{P}<0.05)$, whereas MDA-MB-231 cells transfected with the miR-124-3p inhibitor were significantly more migratory compared with the control cells $(\mathrm{P}<0.05)$ (Fig. 2B). In addition, a Transwell invasion assay was performed to determine the invasive ability of $\mathrm{BC}$ cells transfected with miR-124-3p mimics or an inhibitor. Transfection of cells with miR-124-3p mimics significantly decreased the invasion ability of MCF-7 cells compared with the control cells $(\mathrm{P}<0.01)$, whereas transfection with the miR-124-3p inhibitor significantly increased the invasion ability of MDA-MB-231 cells $(\mathrm{P}<0.05)$ (Fig. 2C). These results suggest that miR-124-3p regulates cell motility and that the inhibition of miR-124-3p may promote breast tumor metastasis.

Western blotting analyses were performed to investigate whether the observed changes in cell motility were accompanied by the epithelial-mesenchymal transition (EMT) of $\mathrm{BC}$ cells. The protein level of E-cadherin was increased by miR-124-3p mimics and suppressed by the inhibitor. The 
protein levels of Vimentin and $\mathrm{N}$-cadherin were promoted by inhibitor and decreased in cells transfected with miR-124-3p mimics (Fig. 2D). These results suggest that miR-124-3p inhibits BC cell EMT and tumor metastasis.

PDCD6 is a direct target of miR-124-3p. All three databases (TargetScan, miRDB and microRNA) predicted PDCD6 as a target of miR-124-3p. PDCD6 has been demonstrated to be overexpressed in metastatic ovarian cancer (12) and the 3'-UTR of PDCD6 mRNA possesses a highly conserved sequence (position 477-484) that is complementary to the seed sequence of miR-124-3p (Fig. 3A). To validate that PDCD6 is a direct target of miR-124-3p, luciferase reporter vectors containing the binding site of WT PDCD6 3'-UTR or the mutant type 3'-UTR were constructed and transfected into MCF-7 and MDA-MB-231 cells along with miR-124-3p mimic or mimic control. The overexpression of miR-124-3p significantly reduced the activity of the WT PDCD6 3'-UTR $(\mathrm{P}<0.05)$, but the activity of the mutant 3'-UTR was unchanged (Fig. 3B). In addition, overexpression of miR-124-3p in MCF-7 and MDA-MB-231 cells reduced the protein expression of PDCD6 (Fig. 3C). These results indicate that miR-124-3p directly targets PDCD6 and inhibits its expression.

Restoration of PDCD6 expression rescues the effect of miR-124-3p on BC cells. In order to demonstrate the functional connection between miR-124-3p and PDCD6, a PDCD6 vector lacking the PCDC6 3'-UTR (PCDC6-no 3'UTR) was transfected into MCF-7 cells stably expressing miR-124-3p. Protein expression levels of PDCD6, and the invasion ability of MCF-7 cells, were restored $48 \mathrm{~h}$ after transfection (Fig. 4A and B). The subsequent reintroduction of PDCD6 with an intact 3'UTR partly restored the inhibitory effect of miR-124-3p mimics on the invasion ability of MCF-7 cells (Fig. 4B), indicating that PDCD6 acts as a functional target of miR-124-3p in BC cells.

miR-124-3p expression negatively correlates with PDCD6 expression in BC tumors. The expression of PDCD6 in primary tumor tissue and adjacent non-tumor tissue from patients with $\mathrm{BC}$ was quantified using RT-qPCR. The mRNA expression level of PDCD6 was significantly increased in primary tumors in comparison with adjacent non-tumor tissues $(4.334 \pm 1.779$ vs. $3.600 \pm 1.750 ; \mathrm{P}=0.001$; Fig. $4 \mathrm{C}$ ). A Spearman's rank correlation test revealed that the expression of miR-124-3p was inversely correlated with PDCD6 expression level (Fig. 4D), indicating that the miR-124-3p/PDCD6 signaling axis serves a role in $\mathrm{BC}$ progression and may be a potential target of novel treatments for BC.

\section{Discussion}

Previous studies have demonstrated that the dysregulation of miRNA contributes to carcinogenesis by suppressing target gene expression; therefore, miRNAs could serve as effective biomarkers for the prognosis and treatment of patients with BC $(13,14)$. Data from the present study demonstrated that miR-124-3p inhibits cell motility and EMT, and that decreased miR-124-3p expression correlated with lymph node metastasis in patients with $\mathrm{BC}$. In addition, PDCD6 was revealed to be a direct target of miR-124-3p. miR-124-3p inhibited the expression of PDCD6 in the BC cells in vitro and PDCD6 expression was negatively correlated with miR-124-3p expression in BC tissues.

In the present study, the expression of miR-124-3p in BC tumor tissue was compared with that in matched normal tissue. The levels of miR-124-3p were significantly reduced in tumor tissue compared with matched normal tissue, which was consistent with previous studies (15-17). These results suggest that the dysregulation of miR-124-3p is associated with the pathogenesis of BC. In addition, downregulation of miR-124-3p was correlated with lymph node metastasis and decreased overall survival probability, which is consistent with previous BC studies (16-18) and studies on other types of tumor $(19,20)$. Therefore miR-124-3p is a promising prognostic marker for various tumors.

In vitro analyses in the present study demonstrated that miR-124-3p inhibits EMT and cell motility. In addition, PDCD6 was identified as a direct target of miR-124-3p. Reintroduction of PDCD6 impaired the tumor suppressor role of miR-124-3p by promoting cell invasion. This suggests that the miR-124-3p/PDCD6 signaling axis serves a role in the increased invasiveness of BC cells. Previous studies have demonstrated that that miR-124-3p directly targets $\rho$-associated protein kinase 1 (21), sphingosine kinase 1 (22), forkhead box protein Q1 (23) and talin 1 (24) to inhibit migration and invasion in numerous types of tumor. Therefore, restoring the expression of miR-124-3p may be an effective therapeutic strategy for advanced cancer.

Overall, the mRNA levels of PDCD6, which is highly expressed in mesenchymal tumors (25) and metastatic ovarian cancer (12), were significantly increased in the BC tissues in the present study. To the best of our knowledge, this is the first study to demonstrate that miR-124-3p mediates its effects on $\mathrm{BC}$ metastasis through the inhibition of PDCD6. The results from the present study revealed a negative correlation between PDCD6 and miR-124-3p expression in BC tissues, demonstrating a functional connection between miR-124 and PDCD6 in primary tumors. The findings from the present study further our understanding of the role of miR-124-3p in breast tumorigenesis and demonstrate that the miR-124-3p/PDCD6 signaling axis could be a target of novel treatments for patients with BC.

\section{References}

1. Torre LA, Bray F, Siegel RL, Ferlay J, Lortet-Tieulent J and Jemal A: Global cancer statistics, 2012. CA Cancer J Clin 65: 87-108, 2015

2. Ha R, Chow D and Wynn R: Global trend in breast cancer imaging research 1992-2012: Bibliometric study. AJR Am J Roentgenol 202: 696-697, 2014.

3. Allemani C, Weir HK, Carreira H, Harewood R, Spika D, Wang XS, Bannon F, Ahn JV, Johnson CJ, Bonaventure A, et al: Global surveillance of cancer survival 1995-2009: Analysis of individual data for $25,676,887$ patients from 279 population-based registries in 67 countries (CONCORD-2). Lancet 385: 977-1010, 2015.

4. Ge L, Zheng B, Li M, Niu L and Li Z: MicroRNA-497 suppresses osteosarcoma tumor growth in vitro and in vivo. Oncol Lett 11: 2207-2212, 2016

5. Liu Z, Gersbach E, Zhang X, Xu X, Dong R, Lee P, Liu J, Kong B, Shao C and Wei JJ: miR-106a represses the Rb tumor suppressor p130 to regulate cellular proliferation and differentiation in high-grade serous ovarian carcinoma. Mol Cancer Res 11: 1314-1325, 2013 
6. He J, Zhang W, Zhou Q, Zhao T, Song Y, Chai L and Li Y: Low-expression of microRNA-107 inhibits cell apoptosis in glioma by upregulation of SALL4. Int J Biochem Cell Biol 45: 1962-1973, 2013

7. Shen B, Zhang Y, Yu S, Yuan Y, Zhong Y, Lu J and Feng J: MicroRNA-339, an epigenetic modulating target is involved in human gastric carcinogenesis through targeting NOVA1. FEBS Lett 589: 3205-3211, 2015.

8. Li Y, Xu D, Bao C, Zhang Y, Chen D, Zhao F, Ding J, Liang L, Wang Q, Liu L, et al: MicroRNA-135b, a HSF1 target, promotes tumor invasion and metastasis by regulating RECK and EVI5 in hepatocellular carcinoma. Oncotarget 6: 2421-2433, 2015.

9. Kan H, Guo W, Huang Y and Liu D: MicroRNA-520g induces epithelial-mesenchymal transition and promotes metastasis of hepatocellular carcinoma by targeting SMAD7. FEBS Lett 589 102-109, 2015.

10. Simbrich A, Wellmann I, Heidrich J, Heidinger O and Hense HW Trends in advanced breast cancer incidence rates after implementation of a mammography screening program in a German population. Cancer Epidemiol 44: 44-51, 2016.

11. Livak KJ and Schmittgen TD: Analysis of relative gene expression data using real-time quantitative PCR and the 2(-Delta Delta C(T)) method. Methods 25: 402-408, 2001.

12. Su D, Xu H, Feng J, Gao Y, Gu L, Ying L, Katsaros D, Yu H, Xu S and Qi M: PDCD6 is an independent predictor of progression free survival in epithelial ovarian cancer. J Transl Med 10: 31, 2012.

13. Yang Q, Wang Y, Lu X, Zhao Z, Zhu L, Chen S, Wu Q, Chen C and Wang Z: MiR-125b regulates epithelial-mesenchymal transition via targeting Sema4C in paclitaxel-resistant breast cancer cells. Oncotarget 6: 3268-3279, 2015.

14. Kleivi Sahlberg K, Bottai G, Naume B, Burwinkel B, Calin GA, Børresen-Dale AL and Santarpia L: A serum microRNA signature predicts tumor relapse and survival in triple-negative breast cancer patients. Clin Cancer Res 21: 1207-1214, 2015.

15. Liang YJ, Wang QY, Zhou CX, Yin QQ, He M, Yu XT, Cao DX Chen GQ, He JR and Zhao Q: MiR-124 targets Slug to regulate epithelial-mesenchymal transition and metastasis of breast cancer. Carcinogenesis 34: 713-722, 2013

16. Dong LL, Chen LM, Wang WM and Zhang LM: Decreased expression of microRNA-124 is an independent unfavorable prognostic factor for patients with breast cancer. Diagn Pathol 10 $45,2015$.
17. Li L, Luo J, Wang B, Wang D, Xie X, Yuan L, Guo J, Xi S, Gao J, Lin X, et al: Microrna-124 targets flotillin-1 to regulate proliferation and migration in breast cancer. Mol Cancer 12: $163,2013$.

18. Arabkheradmand A, Safari A, Seifoleslami M, Yahaghi E and Gity M: Down-regulated microRNA-124 expression as predictive biomarker and its prognostic significance with clinicopathological features in breast cancer patients. Diagn Pathol 10: 178, 2015.

19. Li H, Xie S, Liu M, Chen Z, Liu X, Wang L, Li D and Zhou Y: The clinical significance of downregulation of mir-124-3p, mir-146a-5p, mir-155-5p and mir-335-5p in gastric cancer tumorigenesis. Int J Oncol 45: 197-208, 2014.

20. Li X, Yu Z, Li Y, Liu S, Gao C, Hou X, Yao R and Cui L: The tumor suppressor miR-124 inhibits cell proliferation by targeting STAT3 and functions as a prognostic marker for postoperative NSCLC patients. Int J Oncol 46: 798-808, 2015.

21. An L, Liu Y, Wu A and Guan Y: microRNA-124 inhibits migration and invasion by down-regulating ROCK1 in glioma. PLoS One 8: e69478, 2013

22. Zhang H, Wang Q, Zhao Q and Di W: MiR-124 inhibits the migration and invasion of ovarian cancer cells by targeting SphK1. J Ovarian Res 6: 84, 2013.

23. Peng XH, Huang HR, Lu J, Liu X, Zhao FP, Zhang B, Lin SX, Wang L, Chen HH, Xu X, et al: MiR-124 suppresses tumor growth and metastasis by targeting Foxq1 in nasopharyngeal carcinoma. Mol Cancer 13: 186, 2014.

24. Zhang W, Mao YQ, Wang H, Yin WJ, Zhu SX and Wang WC: MiR-124 suppresses cell motility and adhesion by targeting talin 1 in prostate cancer cells. Cancer Cell Int 15: 49, 2015.

25. la Cour JM, Høj BR, Mollerup J, Simon R, Sauter G and Berchtold MW: The apoptosis linked gene ALG-2 is dysregulated in tumors of various origin and contributes to cancer cell viability. Mol Oncol 1: 431-439, 2008.

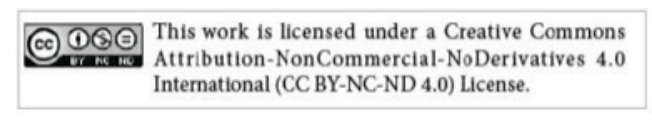

